\title{
Publisher Correction: Does Predictive coding have a future?
}

\author{
Karl Friston (D)
}

Correction to: Nature Neuroscience https://doi.org/10.1038/s41593-018-0200-7, published online 23 July 2018.

In the version of this article initially published, it was not marked as a Historical News \& Views and the supertitle was incorrect. The supertitle should have been "Historical News \& Views: Neural Coding." The error has been corrected in the HTML and PDF versions of the article.

\section{Publisher Correction: Maladaptive cortical hyperactivity upon recovery from experimental autoimmune encephalomyelitis}

Erik Ellwardt, Gautam Pramanik, Dirk Luchtman, Tanja Novkovic, Eduardo Rosales Jubal (1), Johannes Vogt, Isabelle Arnoux @D, Christina Francisca Vogelaar, Shibajee Mandal, Melanie Schmalz, Zeke Barger, Inigo Ruiz de Azua, Tanja Kuhlmann, Beat Lutz, Thomas Mittmann, Stefan Bittner, Frauke Zipp (1) and Albrecht Stroh (10)

Correction to: Nature Neuroscience https://doi.org/10.1038/s41593-018-0193-2, published online 26 September 2018.

In the version of this article initially published, Inigo Ruiz de Azua's name was miscategorized. His given name is Inigo and his surname is Ruiz de Azua. This has been corrected in the HTML coding.

Published online: 7 November 2018

https://doi.org/10.1038/s41593-018-0274-2

\section{Publisher Correction: Enhancers active in dopamine neurons are a primary link between genetic variation and neuropsychiatric disease}

\section{Xianjun Dong (D), Zhixiang Liao, David Gritsch, Yavor Hadzhiev DD, Yunfei Bai, Joseph J. Locascio, Boris Guennewig,} Ganqiang Liu, Cornelis Blauwendraat, Tao Wang, Charles H. Adler, John C. Hedreen, Richard L. M. Faull, Matthew P. Frosch, Peter T. Nelson, Patrizia Rizzu, Antony A. Cooper, Peter Heutink (D), Thomas G. Beach, John S. Mattick (iD, Ferenc Müller (iD and Clemens R. Scherzer (D)

Correction to: Nature Neuroscience https://doi.org/10.1038/s41593-018-0223-0, published online 17 September 2018.

In the version of this article initially published, the legends for Supplementary Figs. 4-8 and 10-14 contained errors. The Supplementary Figure legends have been corrected in the HTML and PDF versions of the article.

\section{Supplementary Figure 4}

Published text: a, Distribution and $\mathbf{b}$, count of protein-coding mRNAs and non-coding (ncRNAs) expressed in dopamine neurons annotated in GENOCDE v19. c, Dopamine neuron transcription factors and the molecular machinery required to produce (dopamine decarboxylase, $D D C$ ), store (vesicular monoamine transporter 2, SLC18A1), and reuptake (dopamine transporter, SLC6A3) dopamine from the synaptic cleft was highly expressed in dopamine neurons (bold gene symbols in $\mathbf{d}$ ). $\mathbf{d}$, Expression of the twenty most abundant mRNAs and g, ncRNAs specific to dopamine neurons (magenta bars) compared to pyramidal neurons (cyan bars) (twenty left-most genes) and vice versa (twenty right-most genes) (note that for each RNA the corresponding bars indicating abundance in the second brain cell type, respectively, are also shown and are close to 0 ; insert in $\mathrm{d}$ ); median \pm m.a.d (median absolute deviation) are shown. $\mathrm{N}=86$ biologically independent dopamine neuron samples and 13 biologgically independent pyramidal neuron samples, respectively. See the full list of neuron-specific genes in Supplementary Table 3. e, The Venn diagram shows the number of mRNAs and h, ncRNAs detected exclusively in dopamine neurons (DA), pyramidal neurons (PY), or non-neuronal cells (NN), respectively. f,i, The twenty abundant, cell type-exclusive mRNAs (f) and ncRNAs (i) were sufficient to accurately cluster the 106 individual samples into dopamine, pyramidal, and non-neuronal clusters. DA, substantia nigra dopamine neurons; MCPY, motor cortex pyramidal neurons; TCPY, temporal cortex pyramidal neurons; PBMC, primary human peripheral blood mononuclear cells; FB, primary human fibroblasts.

Corrected: text: $\mathbf{a}$, Distribution and $\mathbf{b}$, counts of protein-coding mRNAs and non-coding (ncRNAs) expressed in dopamine neurons annotated in GENOCDE v19. c, Dopamine neuron transcription factors and the molecular machinery required to produce (dopamine decarboxylase, $D D C$ ), store (vesicular monoamine transporter 2, SLC18A1), and reuptake (dopamine transporter, SLC6A3) dopamine 